\title{
Gloss and Modelling Studies of Stone Polishing, Using Linear Polishing Machines With Rotating Heads
}

\section{Adriano Manuel Sá Coelho}

Universidade de Lisboa Instituto Superior Tecnico

José Carlos Garcia Pereira

Universidade de Lisboa Instituto Superior Tecnico

Pedro Miguel Gomes Abrunhosa Amaral ( $D$ Pedro.Amaral@tecnico.ulisboa.pt )

Universidade de Lisboa Instituto Superior Tecnico https://orcid.org/0000-0002-6135-8263

Luís Manuel Guerra da Silva Rosa

Universidade de Lisboa Instituto Superior Tecnico

\section{Research Article}

Keywords: ornamental stone, stone polishing, polishing simulation, linear polishing machines

Posted Date: November 2nd, 2021

DOI: https://doi.org/10.21203/rs.3.rs-979709/v1

License: (c) (i) This work is licensed under a Creative Commons Attribution 4.0 International License. Read Full License 


\section{Abstract}

The ornamental stone industry has always played an important role in the world economy, particularly in building construction. Polishing the slabs to increase its gloss, is an important processing operation to enhance the beauty and richness of these natural materials. Many industrial polishing machines rely on rotating heads movement along zigzag trajectories, eroding the surface as stochastically as possible, to avoid scratches and other visual defects caused by paths too symmetric. The displacement of the polishing head after a single zigzag movement and after a single rotation have been used to quantify the polishing process as these two parameters are related with the final stone gloss and are a measure of the efficiency of the polishing process. Applying experimental and new computer simulation techniques, we studied the influence of these two tool displacement parameters on the final stone gloss, and acquired insight into the accuracy of the simulation techniques that were applied here for the first time. We concluded that: 1) a clear correlation can be established between experimental and simulation data; 2) the two displacement parameters represent an effective way to control the quality and efficiency of the polishing process; 3 ) there is a limit for the gloss acquired through polishing processes, so polishing above a given threshold decreases the efficiency without increasing the quality.

\section{Introduction}

The commercial applications of ornamental stones have been progressively focusing on the surface aspect, so the study of the polishing parameters and development of the tools characteristics envisaging the improvement of the surface gloss have an increasing industrial importance, that must be paired with the scientific interest of understanding better the roughness and optical mechanisms that determine these surface phenomena.

Polishing is a finishing process aiming to achieve a high level of surface gloss, through the application of a sequence of abrasives (with decreasing grit sizes) [1-4]. Studying the output produced by the polishing heads (see Fig. 1) on the stone surface allows us to get insight into the influence of the various velocities controlling a linear polishing machine: the conveyor belt velocity, VL, the cross velocity, VT, and the rotational velocity, $\omega$. These studies were carried out measuring the surface gloss (as roughness measurements proved ineffective for the highly polished surfaces that were analysed), and comparing with computer simulations of the same polishing procedures, to better understand the geometrical and kinematic issues determining the final results.

\section{Background}

It has been shown $[1,5,6]$ that the quality and efficiency of a stone polishing process in a linear polishing machine, with one or more rotating heads moving along zigzag trajectories, as indicated in Fig. 2, is controlled by a geometric condition relating the two linear velocities, VL and VT. This condition 1 states that after a complete zigzag movement (with two linear segments), the tool center should be closer to its 
initial position than the tool diameter [1]: $I<d$, with $d=2 r=$ tool diameter. Otherwise there will be gaps of size $\mathrm{I}-2 \mathrm{~d}$ where the polishing head does not interact with the surface.

In this work we suggest that a second condition should be taken into account, relating the linear and rotating tool speeds, thus including all the most relevant kinematic parameters involved in the process.

This condition 2 states that after a complete rotation, the tool center should be closer to its initial position than the tool radius [5]: $x<r$. Otherwise each point of the surface only interacts with a small angular fraction of the circular polishing head, and in some circumstances it may not be touched at all.

The combination of these two conditions determines the relations that must exist between the three velocities involved in this polishing process: the conveyor belt velocity, VL, the transverse head velocity, $\mathrm{VT}$, and the rotational head velocity, $\omega$.

Defining $b$ as the width of the conveyor belt, the time needed for the tool to move across the belt is given by $\mathrm{tT}=\mathrm{b} / \mathrm{VT}$ and the belt shift after a zigzag becomes $\mathrm{I}=2 \mathrm{bVL} / \mathrm{VT} \leq \mathrm{d}$ (limited by condition 1$)$.

The distance XS moved by the polishing head in one linear segment (half of a complete zigzag) is given by:

$$
\mathrm{xS}=\sqrt{\mathrm{b} 2+\left(\frac{\mathrm{l}}{2}\right) 2}=\sqrt{(\mathrm{VTtT}) 2+(\mathrm{VLtT}) 2}=\frac{\mathrm{b}}{\mathrm{VT}} \sqrt{\mathrm{VT} 2+\mathrm{VL} 2}=b \sqrt{1+(\mathrm{VL} / \mathrm{VT}) 2}
$$

Defining $\mathrm{nS}=\omega \mathrm{tT}$ as the number of rotations executed during the time tT by the tool head rotating at $\omega$ rotations per unit of time, the distance $x$ that the tool head moves in a single rotation is given by (limited by condition 2):

$$
\mathrm{x}=\frac{\mathrm{xS}}{\mathrm{nS}}=\frac{b \sqrt{1+(\mathrm{VL} / \mathrm{VT}) 2}}{\omega \mathrm{tT}}=\frac{\mathrm{VT}}{\omega} \sqrt{1+(\mathrm{VL} / \mathrm{VT}) 2} \leq \mathrm{r}
$$

2

To assess the validity of condition 2 and its combination with condition 1, we acquired experimental data and compared with equivalent simulated results.

The polishing operations used in this work involved the last three stages of the polishing sequence (320, 400 and 5Extra grit sequence - see Fig. 3). This sequence was selected to achieve a mirrored surface, with a significant gloss.

a) b) c)

Figure 3 : Sequence of abrasives of the polishing cycle used in this work: a) 320 grit; b) 400 grit; c) 5Extra grit 
The quantitative effectiveness of the surface polishing was then assessed experimentally measuring the gloss with a gloss meter TCQ GL0030 (see Fig. 4), at $20^{\circ}, 60^{\circ}$ and $85^{\circ}$ to the surface normal (the angles supported by the equipment). A wired grid positioned over the stone was used to define each square section where gloss was measured.

The light reflected (the gloss) for a given angle can be compared with a standard scale going from 100 gloss units (GU) for a highly reflecting black glass, to 0 gloss units for a perfectly matt surface [7]. Therefore, the measuring head of a gloss meter must be previously calibrated. Highly reflective materials such as mirrors may achieve gloss values as high as 1000 , when measured at $20^{\circ}$. The measuring angle is selected according to the expected gloss: 1) $20^{\circ}$ for high gloss, above $70 \mathrm{GU}$; 2) $60^{\circ}$ for medium gloss, between 10 and $60 \mathrm{GU}$; 3) $85^{\circ}$ for low gloss, below $10 \mathrm{GU}$. For example, when the gloss measured at $60^{\circ}$ is above $70 \mathrm{GU}$, the measurement should be repeated at $20^{\circ}$ to optimize precision [8].

To quantitatively assess the polishing results, roughness measurements were also tried, but surface fluctuations are too small for common roughness meters. Roughness measurements should be considered in tests involving much coarser surfaces where gloss is too small to be determined with standard equipment.

\section{Computational Details}

A few computer modelling studies have been previously reported $[9,10,11]$ to analyse mechanical and chemical aspects related with the polishing process, but these studies do not aim to simulate the overall macroscopic result of a polishing treatment. To simulate the polishing process, we used PAM - Polishing Analysis Modelling, a new modelling tool developed to simulate the polishing processes occurring in the industry, namely in ornamental stone processing. Users are expected to supply information describing the polishing tools, the operational parameters and the trajectories to follow. In return PAM simulates the polishing process and returns graphic and statistical data allowing users to investigate the results and its causes.

This two-dimensional simulator moves and rotates a tool representation (see Fig. 5) over the stone surface, both represented by lattices of small cells (henceforth designated by pixels, typically with one $\mathrm{mm}$ of side), and accumulates the contacts between them (henceforth designated by abrasion) for each cell. The quality of the polished surface is analysed through a range of parameters that includes, for each pixel, the total abrasion, the $x$ and $y$ average polishing shifts (measured from the pixel to the tool center), the average distance and the distance standard deviation of the pixel (again, relatively to the tool center). Each contact of a surface cell (a pixel) with the polishing tool is accumulated giving the total abrasion. Abrasion can take any value from 0 (black cells) onward, with more white regions representing progressively more eroded cells. Fig. 6 represents the abrasion produced in two simulations of a tool with 320 grit elements, rotating at $600 \mathrm{rpm}$, for 100 and 1000 time steps per second, corresponding to increment angles of 36 and 3.6 degrees, respectively. Fig. $6 a$ shows that, at this rotation speed, a time step of $0.01 \mathrm{sec}$ is clearly insufficient to properly describe the fast movement of the tool over the stone. 
Decreasing the time step to $0.001 \mathrm{~s}$, as shown in Fig. $6 \mathrm{~b}$, the simulator is already able to account for the finer details of the movement, producing an essentially continuous pattern that seems to emulate well a real polishing tool rotating at high speed over a fixed stone.
a) b) c)

a) b)

Figure 6 : Tool head with six 320 grit elements rotating at 10 rotations per second, simulated with: a) a time step of $0.01 \mathrm{~s}$, corresponding to an increment angle of 360 per step; b) a time step of $0.001 \mathrm{~s}$, corresponding to an increment angle of 3.60 per step.

The detailed data that can subsequently be acquired for more realistic simulations, involving complex paths, with straight and curved trajectories, with simultaneous translations and rotations, allow users to get detailed insight into the various aspects governing a stone polishing process.

The quality of the polished surface is analysed through a range of parameters that includes, for each pixel, the total abrasion, the $x$ and $y$ average polishing shifts (measured from the pixel to the tool center), the average distance and the distance standard deviation of the pixel (again, relatively to the tool center). Each contact of a a surface cell (a pixel) with the polishing tool is accumulated giving the total abrasion. The detailed data thus acquired for each simulation allows users to get detailed insight into the various aspects governing a stone polishing process.

\section{Experimental Procedure}

During this work, we used a linear polishing machine with a single head, with a tool diameter $d=435 \mathrm{~mm}$ and a cross distance on a transverse movement $b=240 \mathrm{~mm}$. We used constant values, previously optimised, for the conveyor belt speed $V L=600 \mathrm{~mm} / \mathrm{min}$, the head pressure $\mathrm{P}=2$ bar, and the water flow $\mathrm{Q}=30 \mathrm{~L} / \mathrm{min}$. Six slabs of limestone, as equal as possible, were selected for these tests, due to the high homogeneity of its surface. This is required to simplify the comparison between experimental and simulation data, as the simulator assumes a perfect, two dimensions, stone surface. After applying the sequence of abrasives, the surface gloss is measured and compared with the abrasion predicted in the same conditions by the polishing simulator (see Fig. 7 and Fig. 8). To make the measurements more precise, we used a physical grid made of wire to define precisely the sections where gloss was measured to compare with simulated values. Throughout this work, sections with homogeneous simulated abrasion values were identified as green cells (see Fig. 7) while sections with simulated abrasion values too different were marked red and discarded in the subsequent analysis and comparison with experimental results.

To obey condition $1(\mathrm{l}<\mathrm{d})$, with $\mathrm{VL}=600 \mathrm{~mm} / \mathrm{min}$ as stated above, the transverse speed VT must be VT $\geq 20 \mathrm{~mm} / \mathrm{s}$. To obey condition $2(x<\mathrm{r})$, with $\mathrm{VL}=600 \mathrm{~mm} / \mathrm{min}$ and $\mathrm{VT} \geq 20 \mathrm{~mm} / \mathrm{s}$, the head rotation speed must be $\omega \geq 10 \mathrm{rpm}$. Table 1 shows the various operational conditions tested in this work, whereas 
I values are determined by condition 1 and $\omega$ are determined by condition 2 . Clearly, setting conditions 1 and 2 leads to an increase of the transverse velocity (to decrease I) and an increase of the rotational velocity (to decrease $\mathrm{x}$ ). The simulated linear velocity $(\mathrm{V})$ matches the vectorial combination of both cross and conveyor belt velocities from the polishing machine, according to equation: $\mathrm{V}=\sqrt{\mathrm{V}_{\mathrm{L}}{ }^{2}+\mathrm{V}_{\mathrm{T}}}$.

The parameters used in the $6 \times 4=24$ simulations presented in Tab. 1 aim to replicate the parameters used in the laboratory experiments. The 320, 400 and 5Extra grit abrasives used in this work (see Fig. 3) were simulated and replicated in order to generate the full polishing heads, with six abrasive elements each (see Fig. 5). The simulated stone has the real stone dimensions, assuming pixel cells with $1 \mathrm{~mm} \times 1 \mathrm{~mm}$. The simulated tool paths were designed to emulate the real zigzag trajectories followed by the tool head.

Table 1

24 polishing conditions used in experimental and modelling work, obeying conditions 1 and 2 .

\begin{tabular}{|c|c|c|c|c|c|c|c|}
\hline \multirow[t]{2}{*}{ I (mm) } & \multirow[t]{2}{*}{ VT $(\mathrm{mm} / \mathrm{s})$} & \multirow[t]{2}{*}{$V(\mathrm{~mm} / \mathrm{s})$} & \multicolumn{5}{|c|}{$\omega(\mathrm{rpm})$} \\
\hline & & & $\mathrm{x}:$ & $r$ & $r / 2$ & $r / 4$ & $r / 8$ \\
\hline 120 & 40 & 41.58 & & 10 & 20 & 45 & 90 \\
\hline 64 & 75 & 75.80 & & 20 & 40 & 80 & 165 \\
\hline 53 & 90 & 90.67 & & 25 & 50 & 100 & 200 \\
\hline 48 & 150 & 150.33 & & 40 & 80 & 165 & 330 \\
\hline 32 & 200 & 200.25 & & 55 & 110 & 220 & 440 \\
\hline 24 & 300 & 300.17 & & 80 & 165 & 330 & 660 \\
\hline
\end{tabular}

\section{Results And Discussion}

Given the typical conveyor belt speed used throughout this work ( $\mathrm{VL}=600 \mathrm{~mm} / \mathrm{min}=1 \mathrm{~cm} / \mathrm{s})$ and the relatively large tool head diameter $(\mathrm{d}=435 \mathrm{~mm})$, condition 1 is easily obeyed, even for small transverse speeds (see Tab.1). As expected, increasing the transverse speed requires larger rotational speeds, to fulfil condition 2. Decreasing $x$, from $x=r$ to $x=r / 8$, to further obey condition 2 , requires even larger rotational speeds.

Experimental results (see Fig. 9) for surface gloss as a function of transverse velocity VT, for $x=r, x=r / 2$, $x=r / 4, x=r / 8$ conditions, show a significant increase in gloss from $40 \mathrm{~mm} / \mathrm{s}$ to $200 \mathrm{~mm} / \mathrm{s}$ for all four $X$ conditions. For higher transverse velocities, gloss remains essentially constant, showing that a maximum threshold has been attained and further increasing the energy spent in the polishing process (by increasing the transverse and rotational speeds) does not lead to a refinement of the surface quality. 
The results clearly show that a better surface quality is obtained for smaller $x$ values, when the rotating action is more effective. For $x=r$, the maximum attainable gloss is below $65 \mathrm{GU}$ while for $x=r / 2, x=r / 4$, $x=r / 8$ the maximum gloss is above $70 \mathrm{GU}$, essentially the same value in the three cases. However, for $x=$ $r / 8$ this limit is reached sooner, for lower transverse (and rotational) speeds.

Simulation results (see Fig. 10) for the same conditions show the same general trends, although the flat region for higher transverse velocities is not observed because the simulator simply adds abrasion contacts between the tool and the stone in a linear way, without considering any asymptotic effects.

Clearly gloss increases with transverse velocity, with a slope that essentially matches the values obtained from the experimental curves and the quality of the polished surface clearly increases from $x=r$ to $x=$ $r / 8$. The curve for $x=r / 8$ was obtained from polished surfaces as those represented in Fig. 11, for three different transverse velocities, showing that as the zigzag trajectories become more parallel when the transverse velocity increases, the polishing intensity and surface homogeneity should also increase.

Figure 11 : Abrasion images from polishing simulation, for three transverse velocities VT : a) $40 \mathrm{~mm} / \mathrm{s}$; ) $90 \mathrm{~mm} / \mathrm{s}$; c) $200 \mathrm{~mm} / \mathrm{s}$, with $x=r / 8$.

Figure 12 compares experimental gloss with simulation abrasion, as a function of transverse velocity, for $x=r / 8$. While the simulated abrasion increases linearly with transverse velocity, experimental gloss shows a less predictable evolution, although the overall trends are similar for both types of data. In both cases the polishing quality increases substantially when the transverse speed increases (and rotational speed, to obey condition 2).

However, the data in Fig. 9 and Table 1 clearly show that increasing separately these two speed parameters is not enough to achieve a good polished surface. Decreasing $x$ from $x=r$ to $x=r / 2$ is crucial to achieve a good polished surface. Decreasing $x$ even further to $x=r / 8$ permits obtaining the same results at lower transverse speeds. In our case, working at $x=r / 8$ with VT $=150 \mathrm{~mm} / \mathrm{s}(\mathrm{w}=330 \mathrm{rpm}$, from Table1) provides essentially the same final quality (Fig. 10) as working at $x=r / 2$ with VT $=200 \mathrm{~mm} / \mathrm{s}(\mathrm{w}$ $=110 \mathrm{rpm}$ ). On the other hand, working at low VT speeds such as $90 \mathrm{~mm} / \mathrm{s}$, a good polished surface is never attained, even for $x=r / 8$ and high rotational speeds $(w=200 \mathrm{rpm})$. Transverse and rotational speeds must be expertly combined to achieve adequate levels of polishing at low energy consumption.

\section{Conclusions}

In this set of experiments, we found that values for transverse velocities below $150 \mathrm{~mm} / \mathrm{s}$ are insufficient to achieve the maximum surface quality and values above $250 \mathrm{~mm} / \mathrm{s}$ are just wasting energy.

As expected in a linear polishing machine, the polishing quality increases with transverse and rotational velocities, until some maximum surface quality is achieved. Increasing even more the transverse or rotational velocities does not lead to further refinements in surface quality. Moreover, these two velocities must be expertly combined to get optimal results. Increasing independently the transverse or rotational 
velocities does not necessarily lead to surfaces with maximum quality (industrial empirical evidence, shows that surface quality can even be reduced).

Conditions $\mathrm{I}<\mathrm{d}$ and $\mathrm{x}<\mathrm{r}$ must be applied to get the best results, thus providing mathematical relations between the three velocities (conveyor belt, transverse and rotational) involved in these processes.

These conclusions were sustained by both experimental and simulation results. Further experiments are required to confirm these conclusions, with other types of stone materials and linear polishing machines.

\section{Declarations}

\section{a. Funding}

The results presented in this paper were obtained in the frame of the project 24535 INOVSTONE 4.0, funded by the European Regional Development Fund (FEDER) through the Alentejo Regional Operational Programme (PO Alentejo). This work was also supported by FCT, through IDMEC, under LAETA, project UID/50022/2020.

\section{b. Conflicts of Interest}

The authors have no relevant financial or non-financial interests to disclose.

The authors have no conflicts of interest to declare that are relevant to the content of this article.

\section{c. Availability of data and material (data transparency)}

All data reported has been disclosed in the manuscript.

\section{d. Code availability}

The research software used in the work is under development and, therefore, not available for general purpose yet.

\section{e. Ethics approval}

Not applicable.

\section{f.. Consent to participate}

All the authors agreed participating together in this work.

\section{g.. Consent for publication}

All of the authors have read and agreed to publish this version of the manuscript.

\section{h. Authors' contributions}


Adriano Coelho: conceptualization, investigation, experimental measurements and data collection; writing - reviewing

José Carlos Pereira: conceptualization, investigation, software development and data collection; writing original draft, reviewing, editing and submission.

Pedro Amaral: methodology, investigation, funding acquisition and project management; writing reviewing, editing and submission.

Luís Guerra Rosa: investigation and project management; writing - reviewing.

\section{References}

1. Amaral PM, Rosa LG, Pinto S, Pozo D (2004) New line of diamond tools raise productivity in polishing stone. Industrial Diamond Review, 3, 33

2. Yang H, Li H, Zhu C, Fang H, Li (2017) A process parameters selection approach for trade-off between energy consumption and polishing quality. International Journal of Computer Integrated Manufacturing, Volume 31, 2018 - Issue 4-5: Smart Energy Efficient Manufacturing.

https://doi.org/10.1080/0951192X.2017.1407875

3. Soares Filho JE, Aurich JC, Sousa FJP, Nascimento RM, Paskocimas CA (2018) Estimation of the minimum material removal thickness during the polishing process of ceramic tiles by laser triangulation. Ceramics International, Volume 44, Issue 5, Pages 4646-4652. https://doi.org/10.1016/j.ceramint.2017.12.032

4. Sani ASA, Sousa FJP, Hamedon Z, Azhari A (2016) Distribution of contact pressure over the surface of ceramic floor tiles during the polishing process. IOP Conf. Ser.: Mater. Sci. Eng. 114012008. http://dx.doi.org/10.1088/1757-899x/114/1/012008

5. Barbosa AR (2014) Experimental validation of the ornamental stone polishing simulator. Dissertation, Instituto Superior Técnico, Universidade de Lisboa

6. Barbosa AR, Coelho A, Fernandes JC, Amaral PM, Rosa LG, Pereira JC (2015) A contribution for an optimization of the polishing quality of stone slabs: simulation and experimental study using a singlehead. November 2015, 3rd International Conference on Stone and Concrete Machining (ICSCM 2015)

7. Damares LS (2010) Determinação de parâmetros do polimento em três tipos de rochas graníticas. Dissertation, Campus de São Carlos, Universidade de São Paulo

8. Yavuz H, Ozkahraman T, Demirdag S (2011) Polishing experiments on surface quality of building stones tiles. Construction and Building Materials April 2011, 25(4):1707-1711, https://doi.org/10.1016/j.conbuildmat.2010.10.016 
9. Ranjan P, Balasubramaniam R, Jain VK (2019) Molecular Dynamics Simulation of Mechanical Polishing on Stainless Steel Using Diamond Nanoparticles. J. Manuf. Sci. Eng. Jan 2019, 141(1): 014504 (4 pages). https://doi.org/10.1115/1.4041914

10. Maekawa K, Itoh A (1995) Friction and tool wear in nano-scale machining - a molecular dynamics approach. Wear, Volume 188, Issues 1-2, September 1995, Pages 115-122.

https://doi.org/10.1016/0043-1648(95)06633-0

11. Qin K, Moudgil B, Park C-W (2004)A chemical mechanical polishing model incorporating both the chemical and mechanical effects. Thin Solid Films, Volume 446, Issue 2, 15 January 2004, Pages 277286, https://doi.org/10.1016/j.tsf.2003.09.060

\section{Figures}

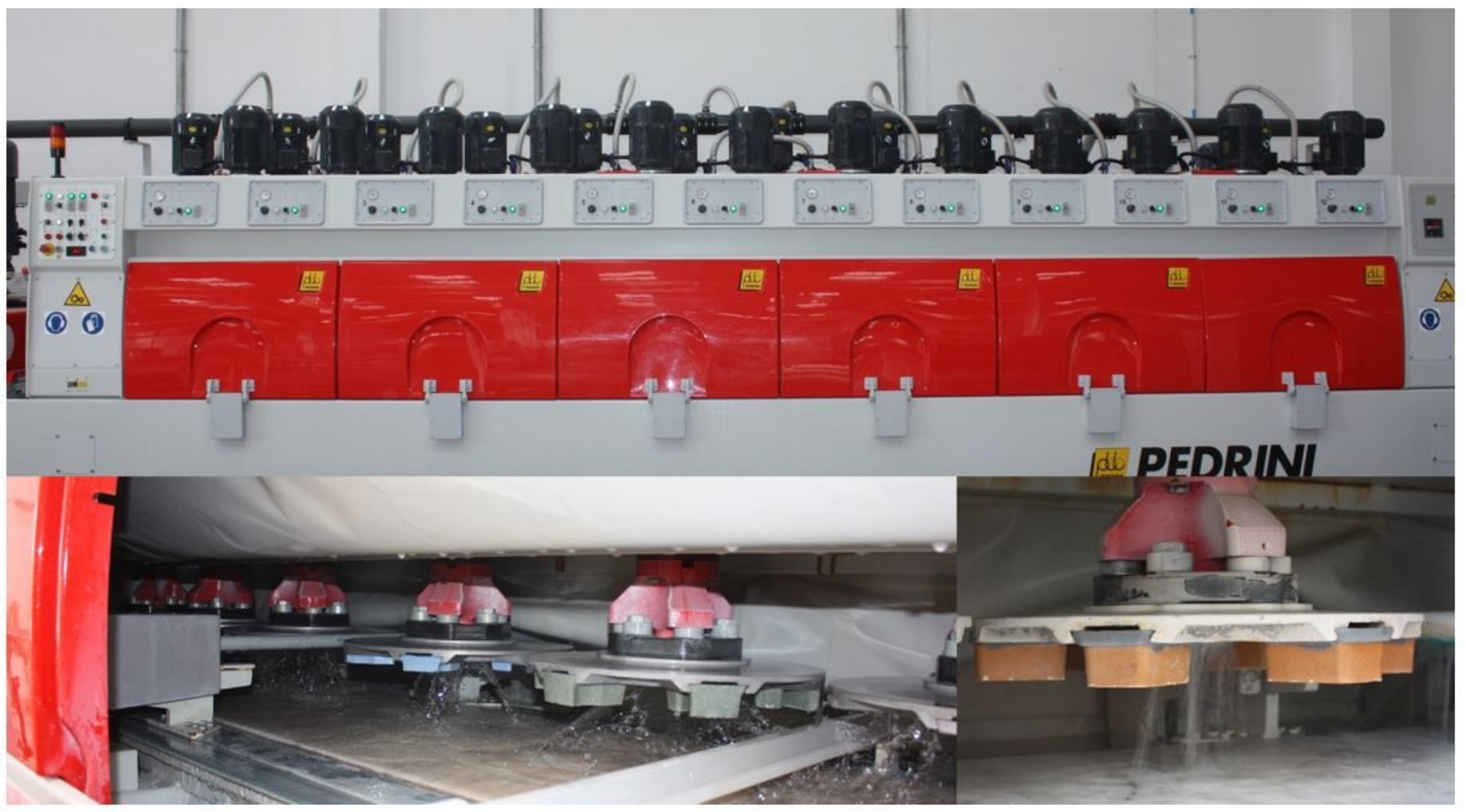

Figure 1

Linear polishing machines with rotating heads (example from PEDRINI). 

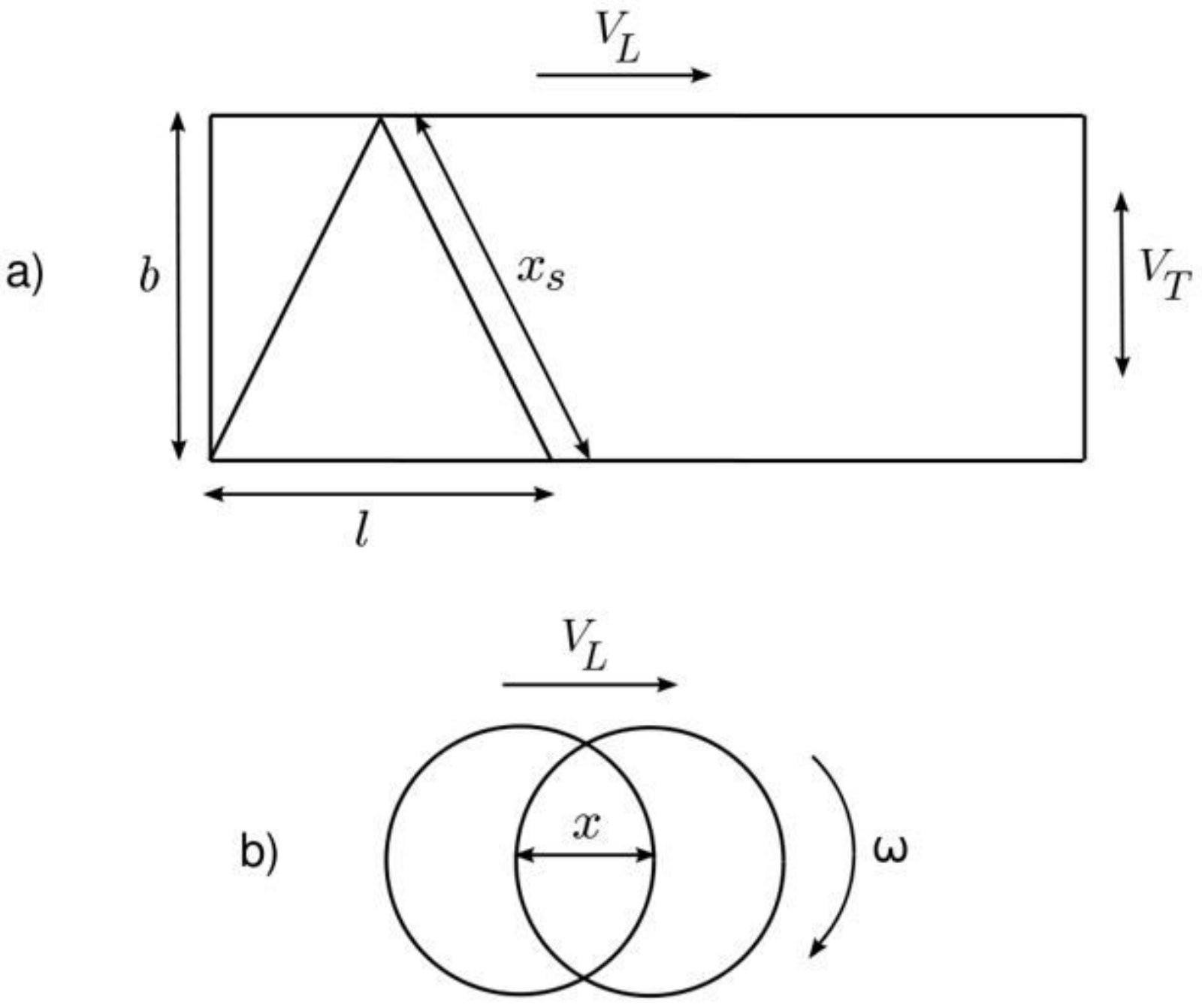

Figure 2

The two polishing conditions. a) $\mathrm{I} \leq \mathrm{d}$; $\mathrm{b}) \mathrm{x} \leq \mathrm{r}$.

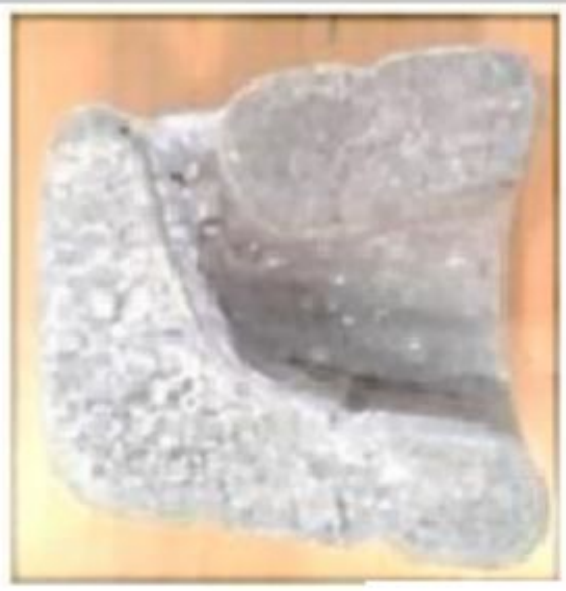

a)

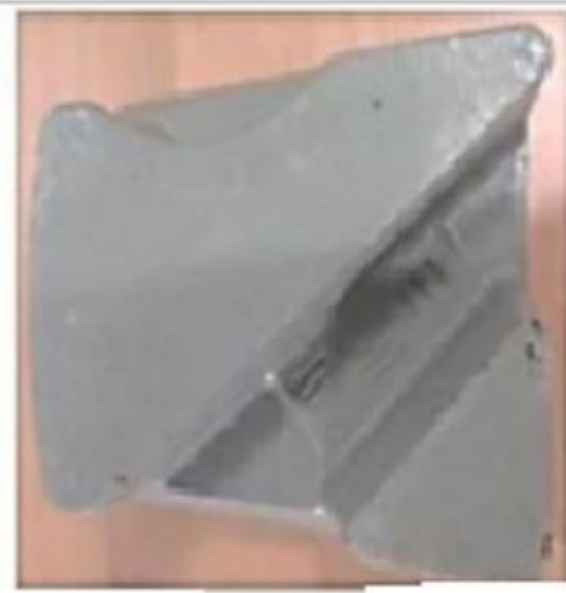

b)

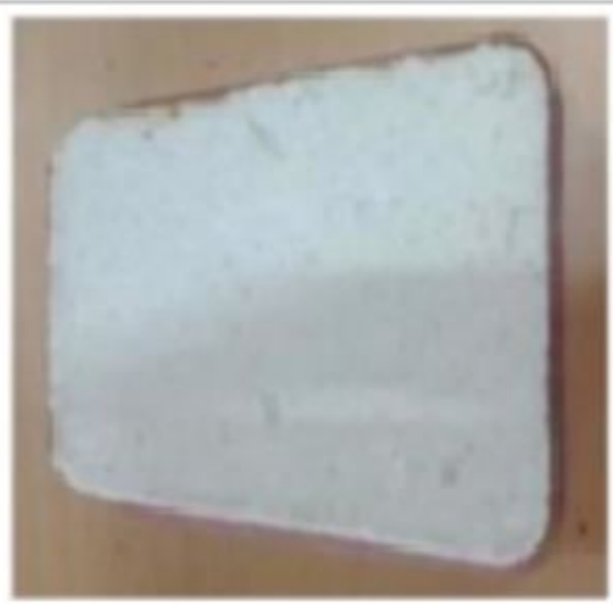

c) 
Figure 3

Sequence of abrasives of the polishing cycle used in this work: a) 320 grit; b) 400 grit; c) 5 Extra grit

\section{Glossmeter TQC GL0030}

Base dimensions: $135 \times 45 \mathrm{~mm}^{2}$

Orifice size:

$50 \times 10 \mathrm{~mm}^{2}$

Spot size :

$20^{\circ}: \pm 5 \times 5 \mathrm{~mm}^{2}$

$60^{\circ}: \pm 20 \times 9 \mathrm{~mm}^{2}$

$85^{\circ}: \pm 30 \times 9 \mathrm{~mm}^{2}$

Resolution:

$0,1 G U(0-100 G U)$ $1 G U(>100 G U)$

Measuring angle characteristics:

$20^{\circ}$

$60^{\circ}$

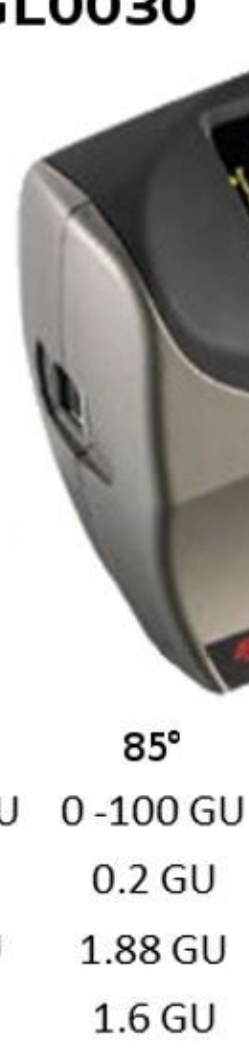

Range

$0-100 \mathrm{GU}$

$0-100 \mathrm{GU}$

$0.4 \mathrm{GU}$

$0.2 \mathrm{GU}$

$1.69 \mathrm{GU}$

$1.58 \mathrm{GU}$

$1.6 \mathrm{GU}$

Reproducibility

$1.2 \mathrm{GU}$

$0.6 \mathrm{GU}$

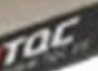

Bias

$1.2 \mathrm{GU}$

\section{Figure 4}

Gloss meter TQC GL0030, used in this work.

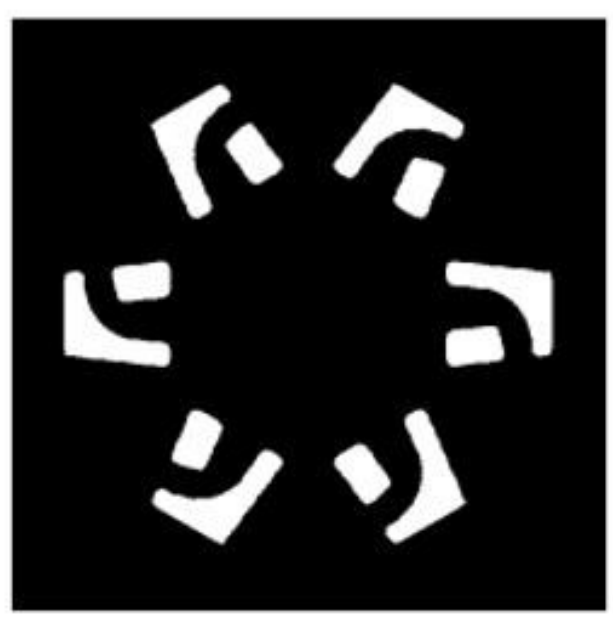

a)

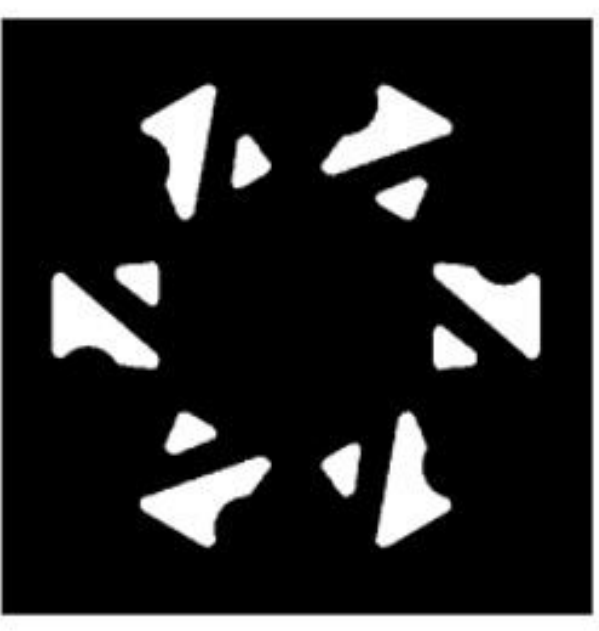

b)

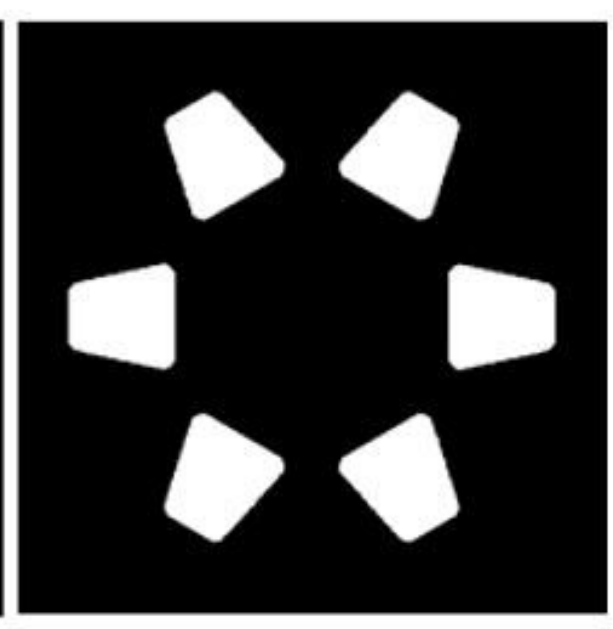

c)

Figure 5 
Simulated tool heads used in this work, containing six abrasive elements of: a) 320 grit; b) 400 grit; c) 5 Extra grit.

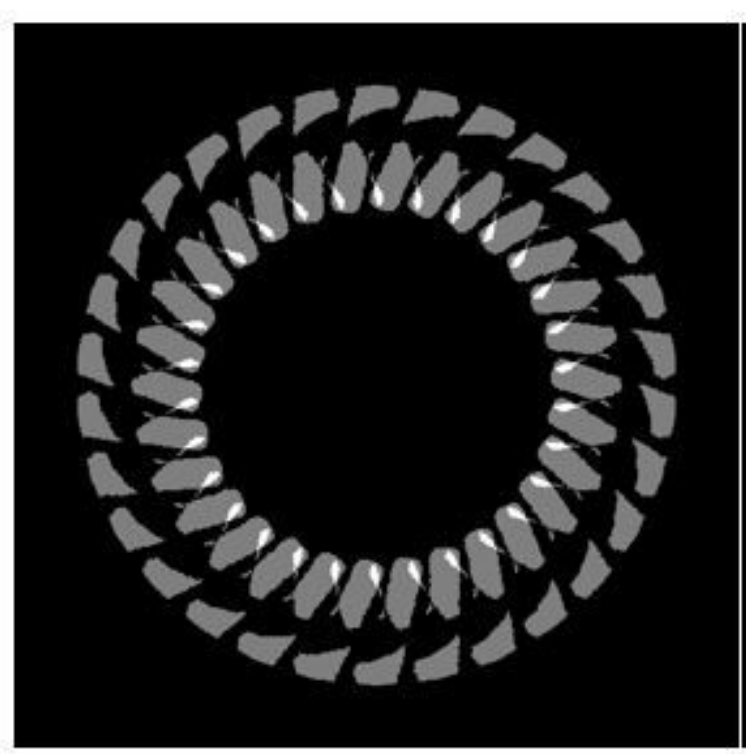

a)

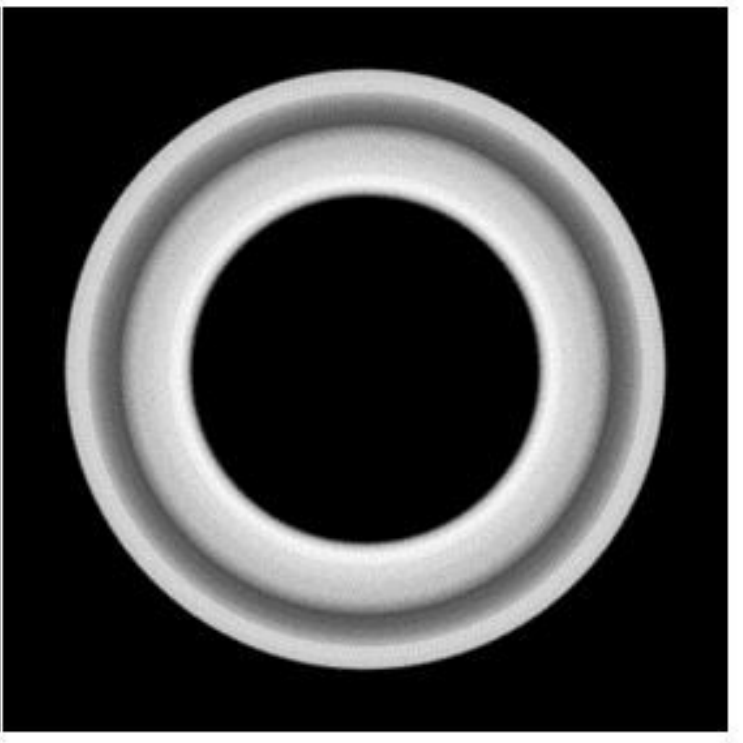

b)

\section{Figure 6}

Tool head with six 320 grit elements rotating at 10 rotations per second, simulated with: a) a time step of $0.01 \mathrm{~s}$, corresponding to an increment angle of 360 per step; b) a time step of $0.001 \mathrm{~s}$, corresponding to an increment angle of 3.60 per step. 


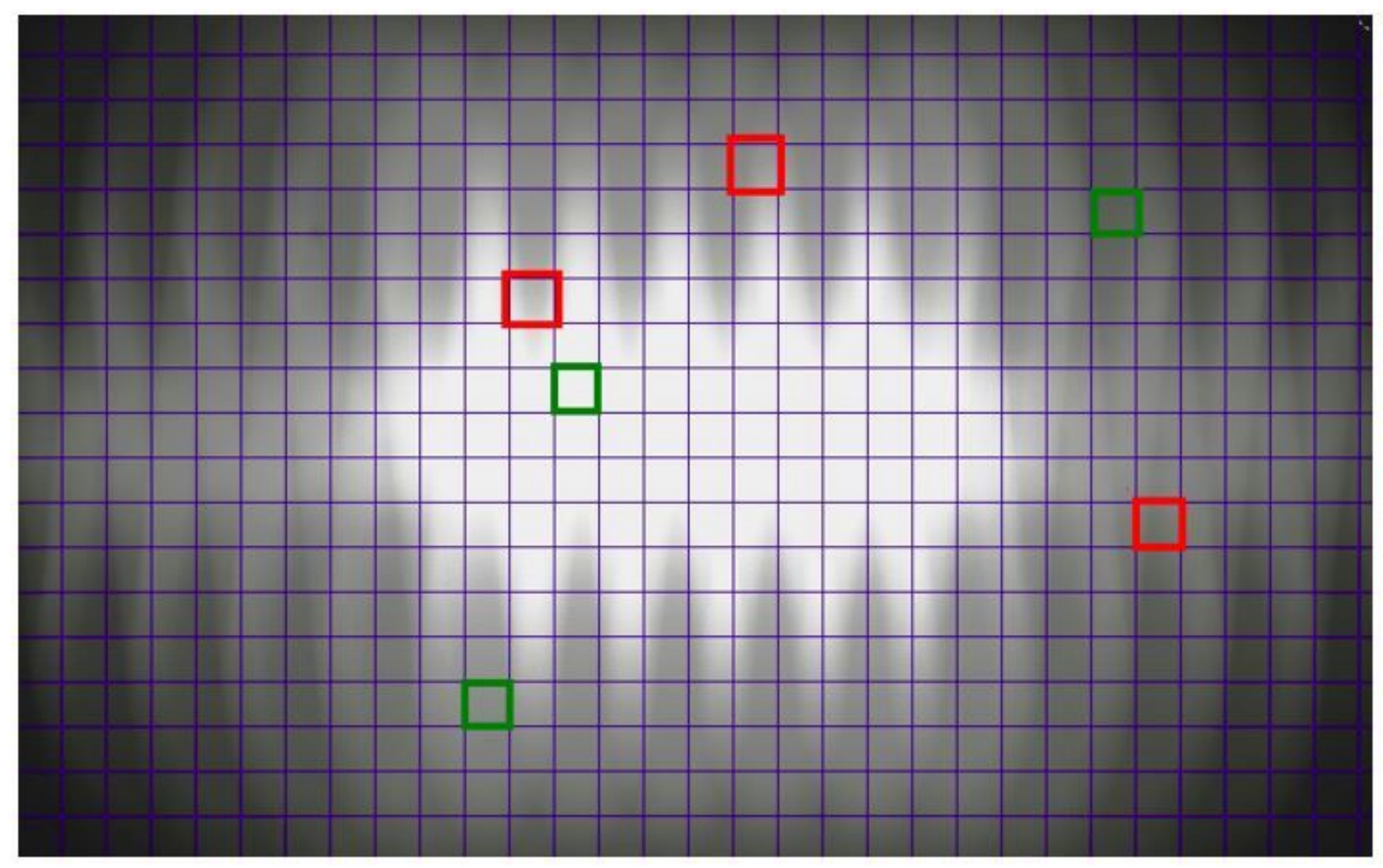

Figure 7

Output image showing the simulated abrasion with homogeneous (green) cells and heterogeneous (red) cells. 


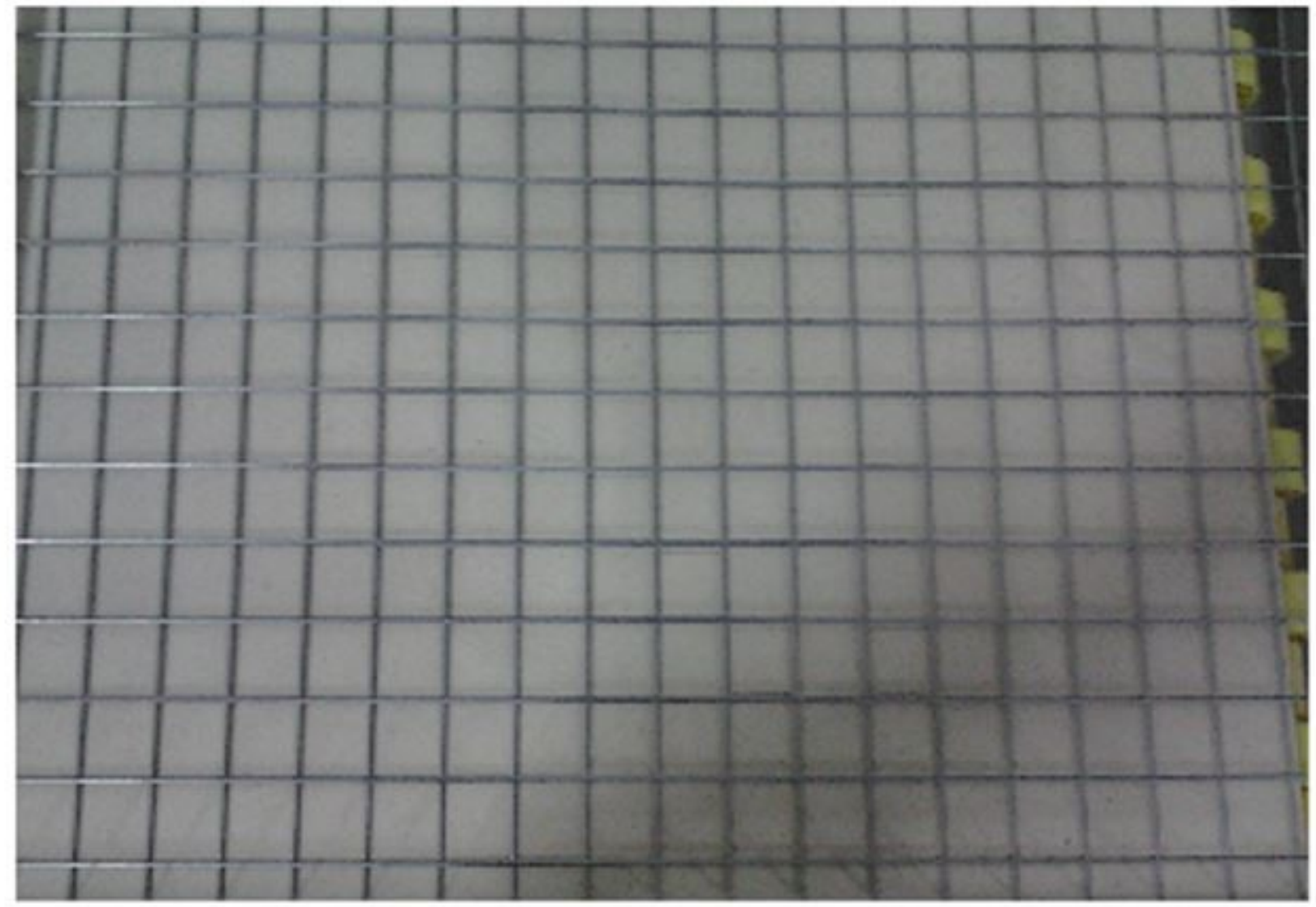

\section{Figure 8}

Experimental setup used to measure gloss data, using a wired grid with cells with $30 \times 30 \mathrm{~mm} 2$, where each gloss measurement takes place.

Tool Dislocation

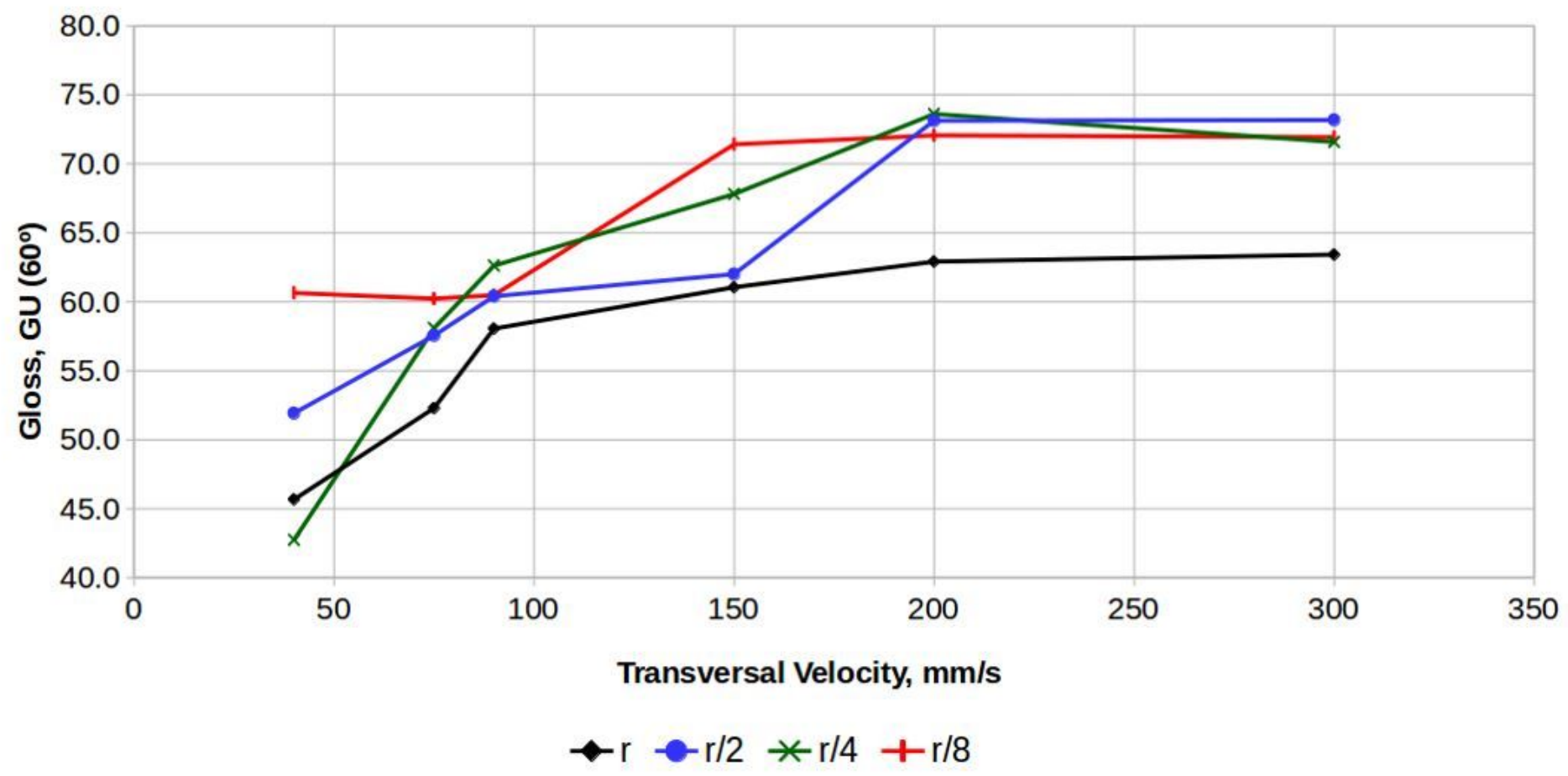




\section{Figure 9}

Gloss as a function of transverse velocity VT, for $x=r, x=r / 2, x=r / 4$ and $x=r / 8$.

\section{Tool Dislocation}

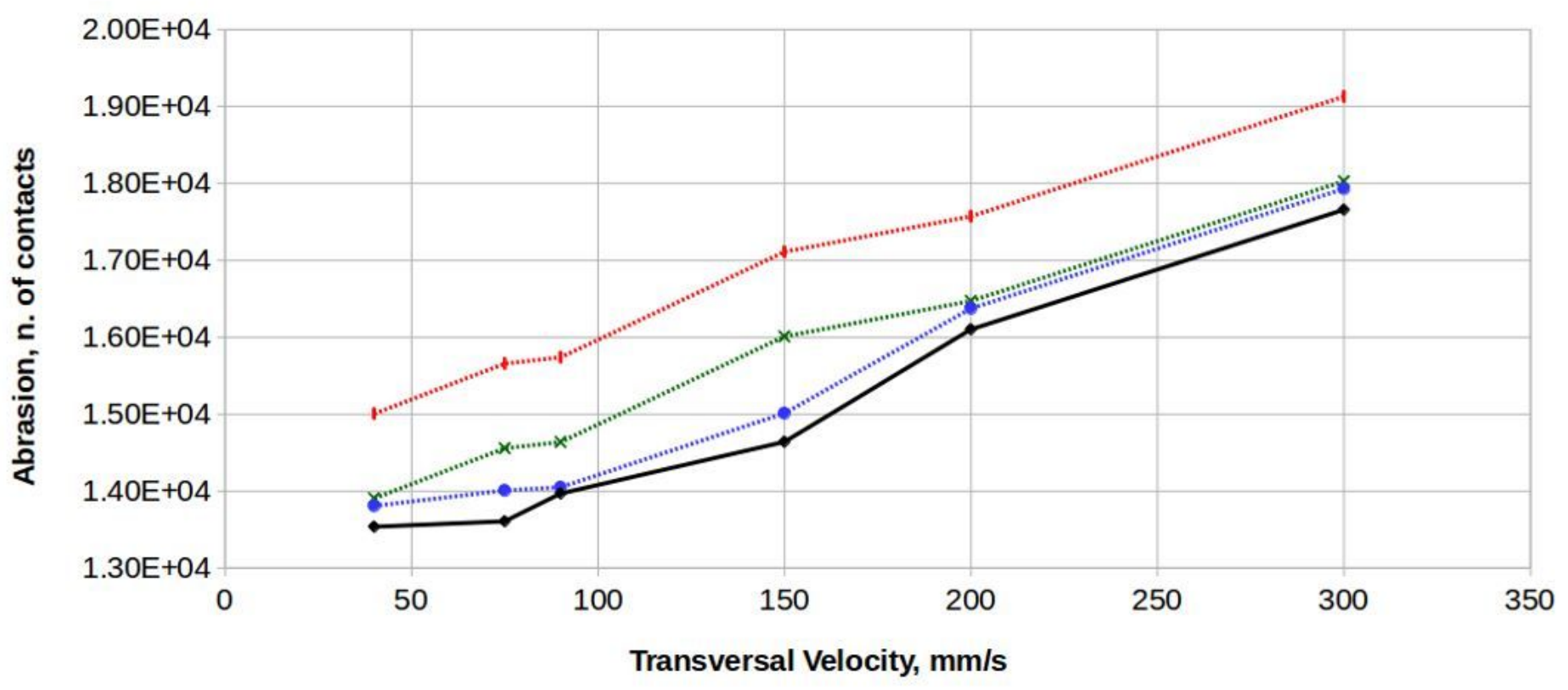

$\rightarrow r \cdots \cdots \cdot r \cdot r / 2 \quad \cdots \cdots \cdot \cdots \cdots \cdot r / 4 \quad \cdots \cdots+\cdots \cdots \cdot r / 8$

\section{Figure 10}

Abrasion as a function of transverse velocity VT, for $x=r, x=r / 2, x=r / 4$ and $x=r / 8$.

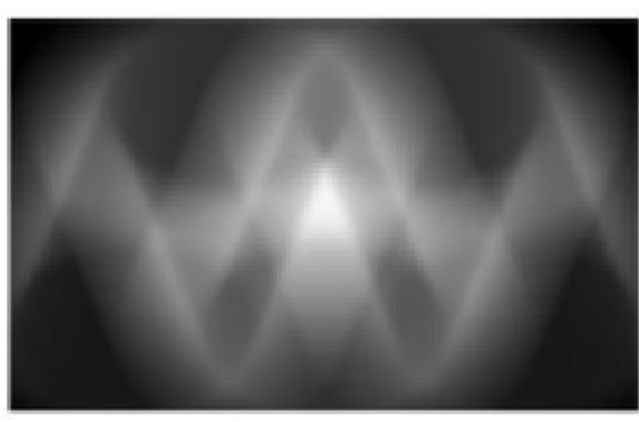

a)

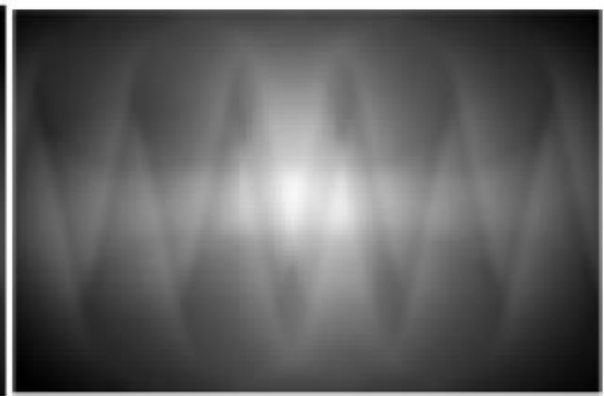

b)

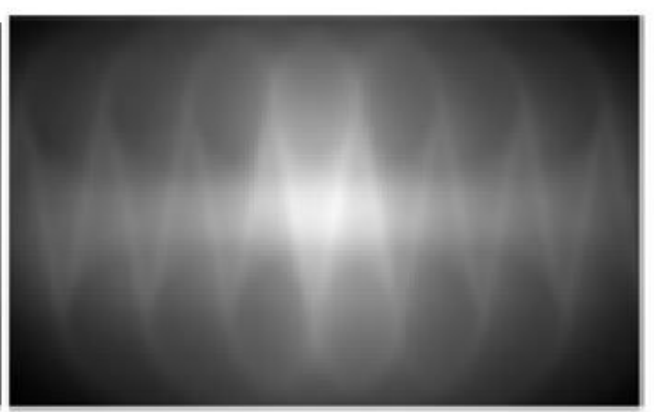

c)

\section{Figure 11}

Abrasion images from polishing simulation, for three transverse velocities VT : a) $40 \mathrm{~mm} / \mathrm{s}$; b) $90 \mathrm{~mm} / \mathrm{s}$; c) $200 \mathrm{~mm} / \mathrm{s}$, with $x=r / 8$. 


\section{Tool Dislocation}

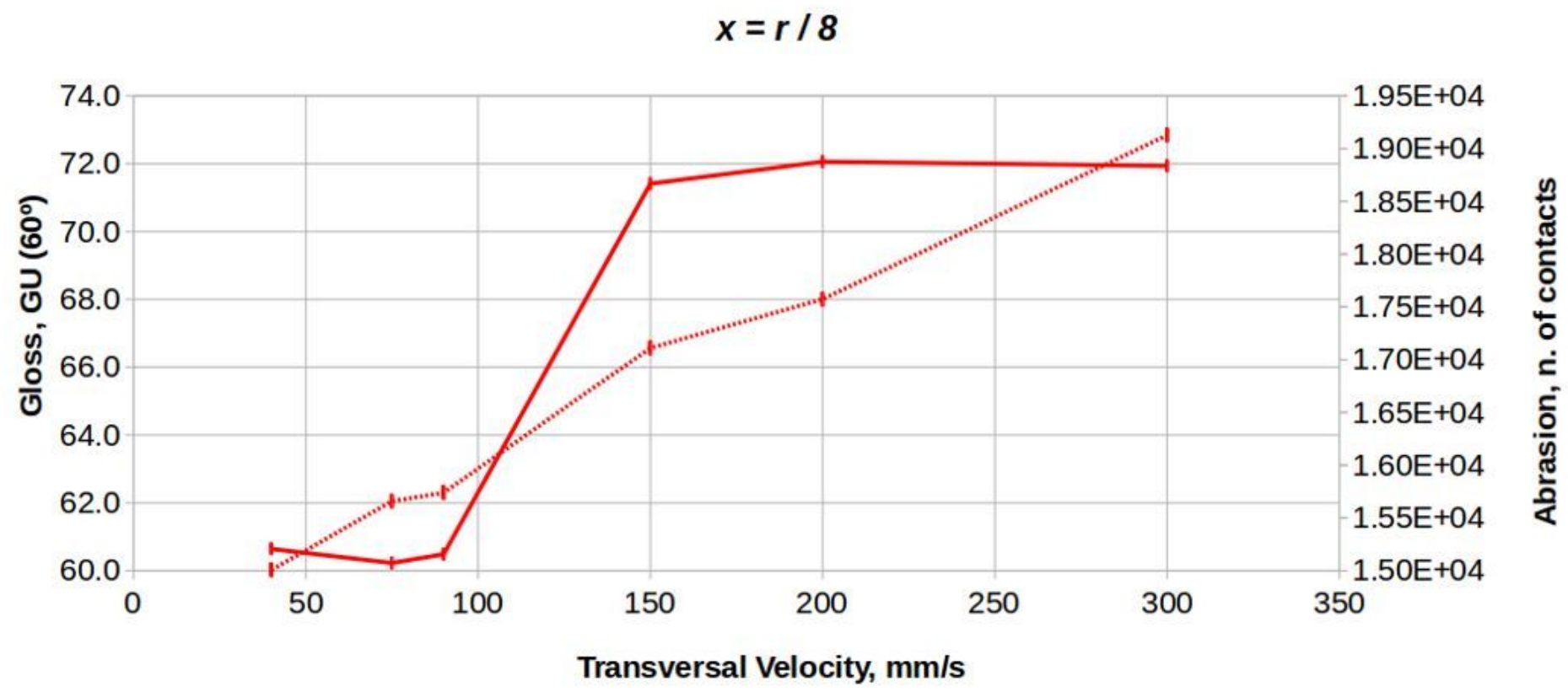

— Gloss Values ............... Abrasion Values

Figure 12

Comparison of abrasion and gloss results, both as in function of transverse velocity VT , for $x=r / 8$. 